Selected Papers of the 6th Polish Symposium of Physics in Economy and Social Sciences FENS2012, Gdańsk, Poland

\title{
Multifractality of Nonlinear Transformations with Application in Finances
}

\begin{abstract}
D. GRech* AND G. PAmula
Institute of Theoretical Physics, University of Wrocław, pl. M. Borna 9, PL-50-204 Wrocław, Poland

We study the multifractal effects of nonlinear transformations of monofractal, stationary time series and apply the found results to measure the "true" unbiased multifractality generated only by multiscaling properties of initial (primary) data before transformations. A difference is stressed between "naive" observed multifractal effects calculated directly within detrended multifractal analysis as the spread $\Delta h$ of the generalized Hurst exponents $h(q)$ and the more reliable unbiased multifractality received after subtraction of residual bias effects generated by nonlinear transformations of initial data and coupled with finite size effects in time series. This property is investigated for volatile series of the real main world financial indices. A difference between multifractal properties of intraday and interday quotes is also pointed out in this context for the Warsaw Stock Exchange WIG index. Finally, based on the observed feature of real nonstationary data, a new measure of unbiased multifractality in signals is introduced. This measure comes from an analysis of the whole generalized Hurst exponent profile instead of looking just at its edge behavior $h^{ \pm} \equiv h(q \rightarrow \pm \infty)$. Such an approach seems to be particularly useful when $h(q)$ is not a monotonic function of the moment order $q$. Interesting examples with extreme events from finance are presented. They convince that an analysis directed only on investigation of the edges $h^{ \pm}$in multifractal spectrum may be misleading.
\end{abstract}

DOI: $10.12693 /$ APhysPolA.123.529

PACS: 05.45.Df, 05.45.Tp, 89.65.Gh, 89.75.Da, 89.75.-k, 89.20.-a, 05.40.-a

\section{Introduction}

Many records of real data from complex systems show the long term persistence. This phenomenon has been studied by many authors in variety of contexts in many diversified areas of science including economics, econophysics and finance. The long-term memory in stationary data of length $L$ is evident as the scaling power law of the two point autocorrelation function $C_{s}=\left\langle\Delta x_{i} \Delta x_{i+s}\right\rangle$ of time series increments $\Delta x_{i}=x_{i+1}-x_{i}(i=1, \ldots, L)$ with the time lag $s[1,2]$ :

$$
C_{s} \simeq(2-\gamma)(1-\gamma) s^{-\gamma}
$$

The autocorrelation scaling exponent $\gamma(0 \leq \gamma \leq 1)$ stands for the level of memory in signal and its two edge values correspond respectively to fully correlated $(\gamma=0)$ or completely uncorrelated $(\gamma=1)$ data.

In the simplest case the scaling law in Eq. (1) has the same unique form for all time scales. One usually refers to such a case as to monofractal data series. The properties of long-term memory in monofractal complex systems are fully described by the main Hurst exponent $H(1 / 2 \leq$ $H \leq 1)[3,4]$ related to $\gamma$ according to [5]:

$$
H=1-\frac{\gamma}{2} \text {. }
$$

However, in many real systems the unique scaling exponent is not sufficient to describe the complex nature of memory in data series. It is a case when fluctuations of different magnitudes follow different scaling laws. Then the correlations between values of the signal also depend on the time scale in which these data are observed. Such systems are called multifractal [6].

Multifractal properties of time series are nowadays studied with particular emphasis because the multiscaling phenomena have been observed in nature in many diversified areas (see below). The accurate estimation of variety of scaling exponents appearing in similar cases is also fundamental to develop suitable simulation or forecasting methods [6].

Among techniques established to quantify multifractal scaling in stationary series one should mention at least two: the wavelet transform modulus maxima (WTMM) method [7] and the multifractal detrended fluctuation analysis (MF-DFA) [8]. The latter one is the $q$-weighted extension of the known DFA method [9, 10] successfully used to evaluation of the Hurst exponent $H$ in monofractal series.

MF-DFA seems to have some advantages over WTMM. The latter method was proven to lead to biased outcomes indicating so-called spurious multifractality [11]. An application of MF-DFA may cover many different problems in science, just to mention examples in seismology [12, 13], cosmology [14], biology [15, 16], meteorology [17], medicine [18], epidemiology [19], music [20, 21], geophysics [22] and mostly finances [23-31].

The "ordinary" fluctuation function $F^{2}(\tau)^{\dagger}$ is replaced in this method by its $q$-th moment $F^{2}(\tau, q)(q \in \boldsymbol{R})$ de- 
fined as follows:

$$
F(\tau, q)=\left\{\frac{1}{2 N} \sum_{k=1}^{2 N}\left[\hat{F}^{2}(\tau, k)\right]^{q / 2}\right\}^{1 / q}
$$

for $q \neq 0$, and

$$
F(\tau, 0)=\exp \left(\frac{1}{4 N} \sum_{k=1}^{2 N} \ln \left(\hat{F}^{2}(\tau, k)\right)\right)
$$

for $q=0$, where

$$
\hat{F}^{2}(\tau, k)=\frac{1}{\tau} \sum_{j=1}^{\tau}\left\{x_{(k-1) \tau+j}-P_{k}(j)\right\}^{2} .
$$

Here $L$ is the total length of signal, $N=[L / \tau]$ stands for the number of non-overlapping boxes, each of size $\tau$ for which detrending procedure is performed and $P_{k}$ is the polynomial trend fitted to initial data in $k$-th time window and then subtracted from these data. The $q$-th order fluctuation function $F(\tau, q)$ in Eqs. (3), (4) is the average value calculated for all possible segments (time windows) of fixed length $\tau$. It satisfies a power-law scaling

$$
F(\tau, q) \sim \tau^{h(q)}
$$

and thus extends the property known from the ordinary DFA [9].

One obtains in this manner the whole continuous set of the Hurst exponents $h(q)$ called also the multifractal profile of the system. For $q<0$ small fluctuations in a signal are relatively amplified with respect to large fluctuations while for $q>0$ is the opposite. This way the scaling properties for fluctuations of various magnitudes are revealed. A commonly accepted measure of multifractality is the spread $\Delta h=h^{-}-h^{+}$, with $h^{ \pm}=h( \pm \infty)$. This spread describes a difference between scaling exponents in Eq. (6) for the smallest and largest fluctuations in series. The $h(q)$ multifractal profile is expected to be a decreasing monotonic function of $q$ for stationary signals [8]. Basically it reflects the fact that smaller fluctuations are more frequent than larger ones in a stationary signal. Hence $\Delta h$ is noticeably positive for multifractals, once for monofractal signals one obtains $\Delta h \simeq 0$.

Since DFA is proven to perform well even for nonstationary series [32], many authors use MF-DFA to analyze multifractal properties of real financial data though we are aware about their nonstationarity. Moreover, the real series provide us with the finite amount of data which leads to finite size effects (FSE) affecting their multifractal shape [33-37].

Recently, it has been shown that a contamination of the initial signal by an additive small amount of white or color noise, short-term memory or periodic data may significantly change the observed multifractal properties of the signal and its corresponding $\Delta h$ spread [38-40]. These effects, called often spurious multifractality, make difficult or even misleading the final interpretation of quantitative results based only on $\Delta h$ values. Therefore the subtle problem of distinguishing between "true" and "apparent" multiscaling phenomena is still a big challenge to face with. This paper makes a contribution to research within that context.

The aim of the presented study is to make an extension of previous findings regarding the influence of FSE amplified by long-term memory [33-35] and the influence of specific nonlinear transformations of data [41] on the observed multifractality. Such nonlinear transformations are often met, e.g. in finance where various volatile time series, i.e. series with lost memory about the sign in data, are investigated together with the primary series of stock prices. Often we are given only transformed data and from their multifractal properties we are forced to conclude about multiscaling properties of primary data before transformation.

We try to answer in this elaboration the following main questions. How much multifractal bias in these primary or volatile series, connected with FSE or with the effect of nonlinear transformations coupled with FSE, can be expected? How can one distinguish if an observed multifractality in transformed series comes as a result of multifractality present already in the beginning in non-transformed data or is just a result of nonlinear transformations done on data without initial multifractal origin? Is it possible to change somehow the present description of multifractality, done so far in terms of $\Delta h$, to include in more reliable way the influence of nonstationarities in data?

In the next section we argue why the bias in multifractality caused by FSE itself or by volatile effects coupled with FSE is important even for stationary artificially constructed data. Then an analysis of such effect for examples of real financial time series is investigated in some details in Sect. 3 in variety of scenarios. We show therein an importance of multifractal bias in proper determination of multifractal properties of real nonstationary systems. A new proposal connected with an alternative description of multifractality within MF-DFA scheme which seems to overcome difficulties of spurious effects in real series is introduced in Sect. 4.

\section{Multifractal bias effects in artificial stationary series of data and their nonlinear transformations}

We start with an academic example of monofractal and multifractal artificially created time series in order to pay attention at some properties of their observed multifractal structure. These properties will be discussed and compared in the following sections with the similar effects found in real data mined from financial market. The observed structure is usually the superposition of various effects influencing or generating multifractal property of a given complex system. An important part of this multifractal picture is the bias (called sometimes also multifractal noise) generated by finite length of data amplified by the long-term memory in a system and nonlinear transformations done on these data. The latter transformations change the main Hurst exponent value and 
the generalized Hurst exponents $h(q)$, generating multifractality in transformed data even if they were initially monofractal. In the simplest case this modification can be accessed analytically [41]. However, a quantitative description in a general case of nonstationary data or for more complicated volatile transformations makes a big challenge.

We will consider the following nonlinear "volatile" transformations of initial primary data series $\Delta x_{i}$ :

$$
\begin{aligned}
& \text { 1. } x_{i} \rightarrow u_{i}^{(1)}=\left|\Delta x_{i}\right|, \\
& \text { 2. } x_{i} \rightarrow u_{i}^{(2)}=\left(\Delta x_{i}\right)^{2}, \\
& \text { 3. } x_{i} \rightarrow\left|r_{i}\right|=\left|\frac{\Delta x_{i}}{x_{i}}\right|, \\
& \text { 4. } x_{i} \rightarrow \mu_{i}=(1 / s) \sum_{k=i-s+1}^{i}\left|r_{i}\right|, \\
& \text { 5. } x_{i} \rightarrow v_{i}=\left[(1 / s) \sum_{k=i-s+1}^{i}\left(r_{k}-\left\langle r_{k}\right\rangle_{s}\right)^{2}\right]^{\frac{1}{2}},
\end{aligned}
$$

where $\left\langle r_{k}\right\rangle_{s}=(1 / s) \sum_{i=k-s+1}^{k} r_{i}$ is the moving average of $r_{i}$ of length $s$. We assumed within this paper $s=21$. They are often used in literature to eliminate the sign in data and define various types of less or more complicated volatility series discussed in econophysics and in economics. Let us note that transformations (1) and (2) are used interchangeably as the simplest model of volatility [41], (3) describes the series of absolute returns in finances, (4) stands for the moving average of absolute returns of length $s$, and the last transformation (5) is a typical volatility definition used by financiers.

We started with a construction of artificial monofractal and multifractal time series of fixed length $L=2^{16}$, to examine how their observed multifractal properties change with different nonlinear transformations (1)-(5) applied to primary signal data $u_{i}^{(0)}=\Delta x_{i}(i=1, \ldots, L)$. The monofractal series were generated by the Fourier filtering method (FFM) [42] while the multifractal series have been mimicked by binomial cascade algorithm [43].

First, we revealed relations between the main Hurst exponents $H$ before transformation and after each of transformations (1)-(5). They are shown in Fig. 1 (top) for monofractal case and in Fig. 1 (bottom) for binomial cascade model. In the latter case the $H$ exponent is uniquely expressed by $a\left(\frac{1}{2}<a<1\right)$ parameter used to generate consecutive steps in the algorithm (see [43] for details):

$$
H=\frac{1}{2}\left[1-\log _{2}\left(a^{2}+(1-a)^{2}\right)\right] .
$$

Let us note that Fig. 1 (top) makes an extension of the dependence between $H$ values for primary series $u_{i}^{(0)}$ and $u_{i}^{(2)}$ shown in [41] (see Fig. 1 therein). Other nonlinear transformations modify also remarkably the persistency of transformed series - both in monofractal and multifractal cases.

The nature of dependences in Fig. 1 indicates that multifractal profiles $h(q)$ for these series after transformations might be also seriously modified. An example of multifractal profile for binomial cascade of length $L=2^{16}$

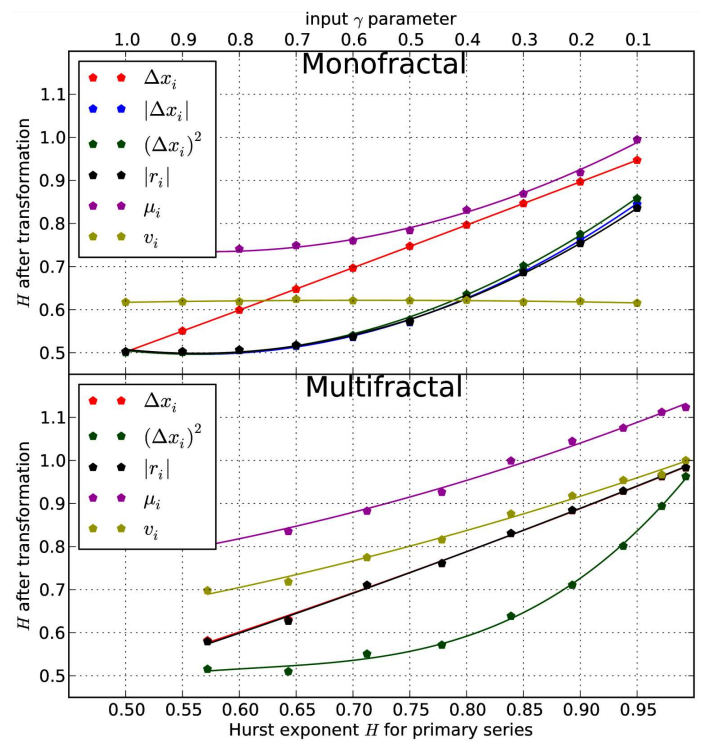

Fig. 1. Dependence between the main Hurst exponent $H$ before and after nonlinear transformation obtained for artificial data of length $L=2^{16}$. Top figure shows the result for monofractal series and the bottom one for multifractal case. See main text for explanation of used symbols.
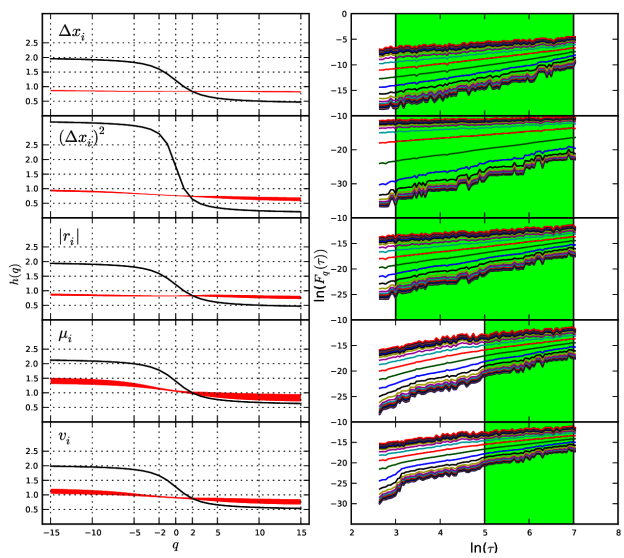

Fig. 2. Multifractal properties of artificially created multifractal time series in binomial cascade model $(a=$ $\left.0.75, L=2^{16}, H=0.84, \gamma=0.32\right)$. Left column presents multifractal profile (black curve) and the corresponding total multifractal bias at $95 \%$ confidence level (red). Right column shows multiscaling properties for integer moments $q=-15,-14, \ldots, 14,15$ (from the bottom to top) and the used scaling ranges (green).

for $a=0.75(H=0.84)$ is drawn in Fig. 2. We have also indicated here the red ribbon-like area for any $h(q)$ profile. This area will play a central interest in further analysis because it corresponds to the multifractal bias present in all cases and defined as follows. Let us take an ensemble of monofractal series with the same $H$ exponent as the input main $H$ value for the given multifractal series of the same length. Then nonlinear transforma- 


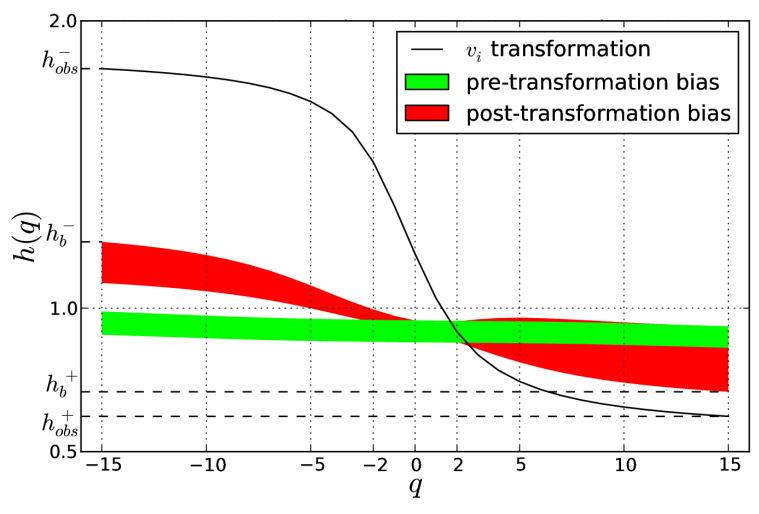

Fig. 3. Example of multifractal $h(q)$ profile (black curve) of transformed artificial multifractal series simulated within binomial cascade model for $a=0.7$ $(H=0.9), L=2^{16}(\gamma=0.22)$. Two biases are indicated: one generated by FSE amplified by long-term memory present in the series (green) and the second accounting the influence of nonlinear $v_{i}$ volatility transformation coupled with FSE (red).

tions on this monofractal series can be performed and the set of multifractal profiles for them can be found at a given (here $95 \%$ ) confidence level. This is clarified in Figs. 2, 3. The red ribbon-like area in these figures covers exactly such multifractal profiles. In other words any profile outside this area has $95 \%$ probability to indicate multifractality in primary data which results not due to the FSE nor due to applied transformations. We will call this multifractality unbiased one, while any eventual $h(q)$ profile within the ribbon area will result from a multifractal bias (at given confidence level). Obviously, according to such a definition, multifractal effects generated for the monofractal signals after applying nonlinear transformations will also be called a multifractal bias. We would like to distinguish it from multifractal effects arising from the amplification of a multifractality existing in primary series itself before the nonlinear transformation is applied. Therefore we have the following relations in terms of the edge $h^{ \pm}$values (see also Fig. 3 for an illustration):

$$
\begin{aligned}
& h_{\mathrm{obs}}^{-}=h_{\mathrm{b}}^{-}+\Delta h^{-}, \\
& h_{\mathrm{obs}}^{+}=h_{\mathrm{b}}^{+}-\Delta h^{+},
\end{aligned}
$$

where $h_{\mathrm{obs}}^{ \pm}$are the observed (biased) edge values of the multifractal profile, $h_{\mathrm{b}}^{ \pm}$are the edge values of multifractal bias (at given confidence level $95 \%$ ), while $\Delta h^{\mp}$ are increments above (below) the multifractal bias level.

\section{Hence}

$$
\Delta h_{\mathrm{obs}}=h_{\mathrm{obs}}^{-}-h_{\mathrm{obs}}^{+}=\Delta h_{\mathrm{b}}+\Delta h_{\mathrm{unb}}
$$

where $\Delta h_{\mathrm{b}}=h_{\mathrm{b}}^{-}-h_{\mathrm{b}}^{+}$is the bias level and $\Delta h_{\mathrm{unb}}=$ $\Delta h^{-}+\Delta h^{+}$is the unbiased spread of generalized Hurst exponents.

To see the qualitative role of biased and unbiased multifractal effects in the discussed volatility transformations we show in Table I the corresponding values of $\Delta h_{\mathrm{obs}}$ and $\Delta h_{\mathrm{unb}}$ for different transformations for the simple
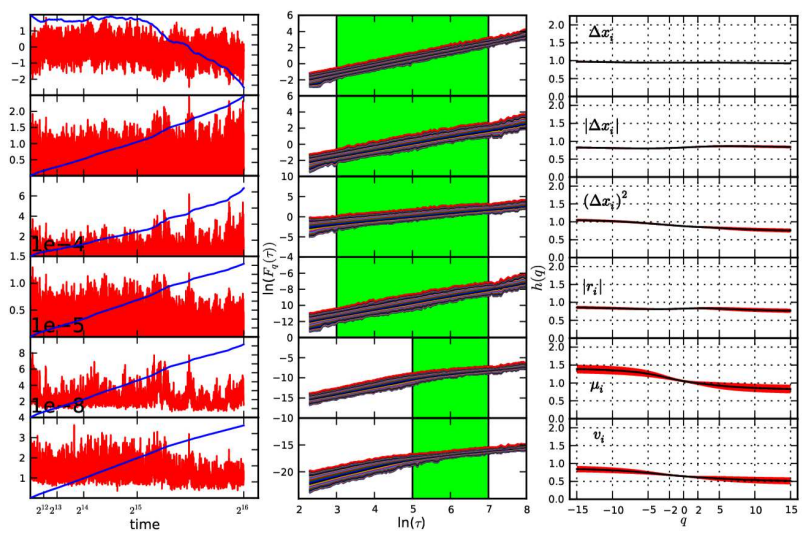

Fig. 4. Results of nonlinear transformations applied to monofractal data with long-term memory $(\gamma=0.1, L=$ $\left.2^{16}\right)$. Left column shows the plots of series increments (red) after particular transformation and the integrated profile of transformed data (blue curve). Middle column indicates multiscaling properties for $-15 \leq q \leq 15$ and the used scaling range (marked in green). Right column contains multifractal profiles $h(q)$ (black) and the statistical uncertainty at $95 \%$ confidence level (red ribbon-like domain) calculated on the ensemble of $10^{2}$ independent simulations in FFM method. It defines global multifractal bias caused together by nonlinear transformation and FSE in data.

TABLE I

Example of observed and unbiased multifractal spread for the simulated multifractal series of length $L=2^{16}$ in binomial cascade model $(a=0.75)$.

\begin{tabular}{c|c|c|c|c|c}
\hline \hline & $\Delta x_{i}$ & $\left(\Delta x_{i}\right)^{2}$ & $\left|r_{i}\right|$ & $\mu_{i}$ & $v_{i}$ \\
\hline$\Delta h_{\text {obs }}$ & 1.49 & 3.08 & 1.47 & 1.49 & 1.45 \\
$\Delta h_{\text {unb }}$ & 1.41 & 2.69 & 1.29 & 0.68 & 0.91
\end{tabular}

binomial cascade model with $a=0.75, L=2^{16}$. It is remarkable that for some transformations (4), (5) the bias effect is quite significant and is responsible for $\approx 18-37 \%$ of the observed multifractality. In other words, only the rest of the effect (unbiased multifractality) is caused by the presence of multiscaling in primary data. When one considers monofractal series (see Fig. 4), the unbiased multifractality obviously disappears. Let us note however that multifractal bias still exists in the latter case due to FSE and applied nonlinear transformations.

\section{Multifractal bias in real primary and volatile financial series of data}

Let us switch now to examples of real time series in finance. They often contain nonstationary data and extreme events like crashes which makes them more interesting from practical point of view. Simultaneously, it is also more difficult to describe. Figure 5 shows the multifractal analysis of S\&P 500 index closure day data in two adjacent time windows: since Nov. 13, 1981 till Oct. 29, 1985 and since Oct. 30, 1985 till Oct. 12, 1989, each 
containing $10^{3}$ data points. The similar investigation of Nikkei225 index data in adjacent windows $(2 \times 1000$ datapoints): since Nov. 27, 2003 till Dec. 19, 2007 and since Dec. 20, 2007 till Jan. 30, 2012 is presented in Fig. 6. An accuracy of fit to Eq. (6) is confirmed by the set of scaling range plots in Fig. 7 showing results for the latter case of Nikkei225 index. The scaling ranges for S\&P500 index were the same and scaling quality similar (not shown). The detrending in MF-DFA was done in all cases with the second degree polynomial trends $P_{k}(i)(k=2)$.
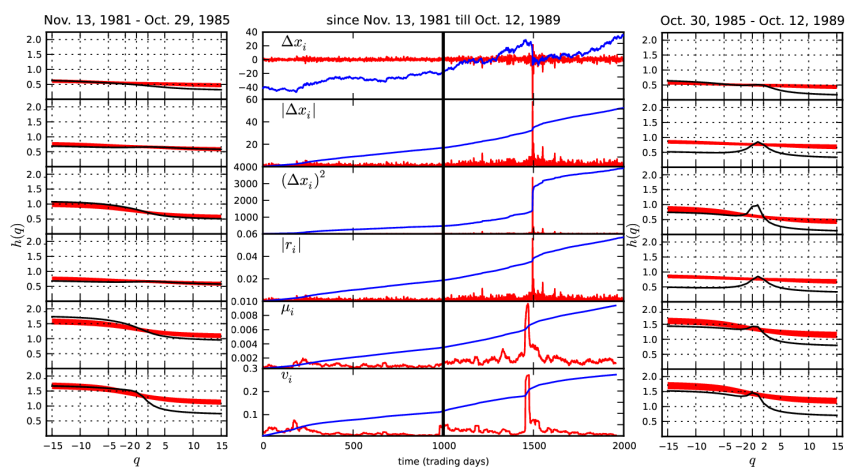

Fig. 5. Multifractal analysis of S\&P500 closing day values and their volatile transformations since Nov. 13 , 1981 till Oct. 12, 1989, separated into two adjacent windows of length 1000 each. Middle-left part describes the steady period on the market from Nov. 13, 1981 Oct. 29, 1985, once the middle-right part contains data from Oct. 30, 1985-Oct. 12, 1989 and includes the famous Black Monday crash of October 19, 1987. Plots in the left and right column show results of multifractal profiles with corresponding full biases resulting from FSE and nonlinear transformation at 95\% confidence level (red) for primary (first row) and transformed data (remaining plots in both columns).
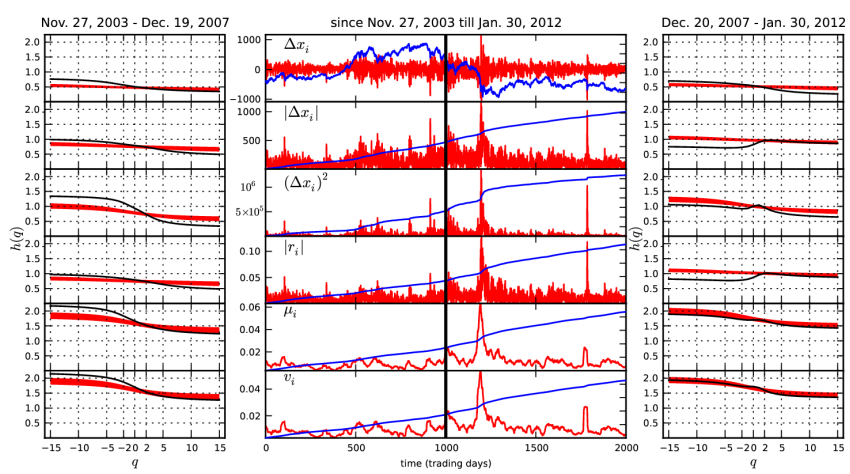

Fig. 6. The same as in Fig. 5 but for Nikkei225 index since Nov. 27, 2003 till Jan. 30, 2012. Data in the left window are more steady, while the right adjacent window contains the great crash of the fall 2008 and the smaller one of March 2011.

Two adjacent time windows in this analysis were not chosen accidentally. Once the first window corresponds to more steady period in stocks (see the left parts in

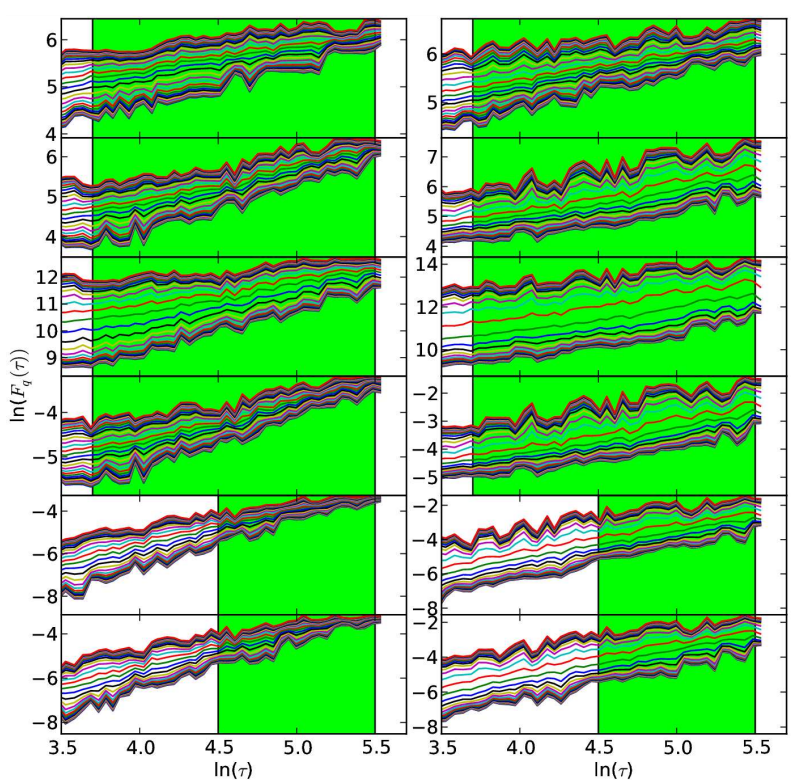

Fig. 7. Multiscaling properties in adjacent windows for real data (Nikkei225 index) from Fig. 6. Plots indicate multiscaling properties for $-15 \leq q \leq 15$ for corresponding parts in Fig. 6 and the scaling range (marked in green) used for calculations of $h(q)$ profiles.

Figs. 5 and 6) the next time window describes much more dynamical situation with at least one extreme event present (see the right parts in Figs. 5 and 6). We will focus on two extreme events - the largest financial crashes in last quarter which already were the subject of interest for economists (see e.g. [44, 45]) and physicists (see e.g. [46-48]). They are respectively the crash of October 19th, 1987, known as the Black Monday, visible in S\&P500 index historical data in Fig. 5 (top middle-right part) and the crash of the fall 2008 indicated in Fig. 6 for Nikkei225 index (top middle-right part). We see that multifractal profiles for primary and transformed data in the first discussed window for Nikkei225 or S\&P500 keep their monotonically decreasing shape independently of the nonlinear transformation (1)-(5) which has been applied to data. It is not the case for the series in the adjacent time window (see the middle-right part columns in Figs. 5 and 6 ). The increments of time series cluster here more intensively and are much more heterogeneous. This property becomes evident particularly for $\left(\Delta x_{i}\right)^{2},\left|\Delta x_{i}\right|$ and $\left|r_{i}\right|$ transformations. They become more smooth in averaging transformations like (4), (5). The richest observed (biased) multifractal structure is found therefore for transformations (2), (3) and for highly nonstationary data containing an extreme event.

Let us notice that the multifractal bias resulting from FSE and from the effect of applied nonlinear transformations, shown as the red ribbon-like area in Fig. 2, increases with the complexity level of transformation, i.e. from (2) to (5). This can cause difficulties in the correct reading of the results from the multifractal profile and 

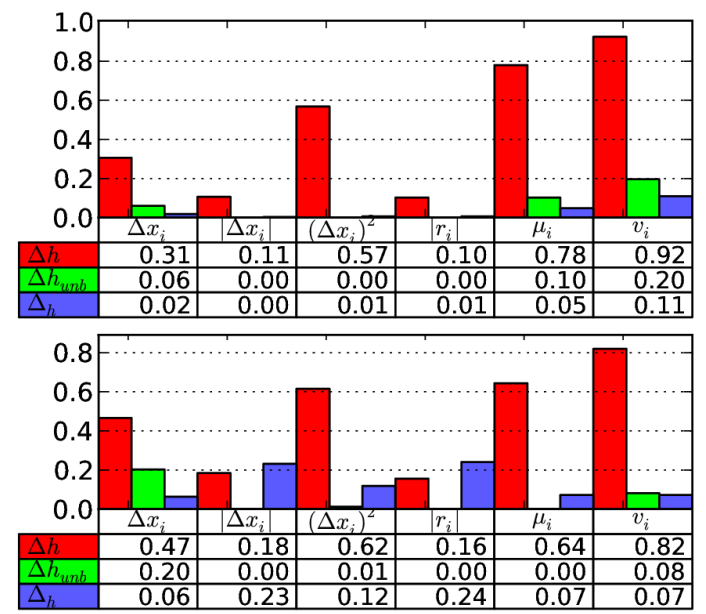

Fig. 8. Comparison of three measures of multifractality: $\Delta h_{\mathrm{obs}}, \Delta h_{\mathrm{unb}}$ and $\Delta_{h}$ for S\&P500 index in two adjacent time windows since Nov. 13, 1981 till Oct. 29, 1985 and since Oct. 30, 1985 till Oct. 12, 1989 (shown are results for the primary data increments $\Delta x_{i}$ and for all nonlinear transformations of these increments discussed in the main text).
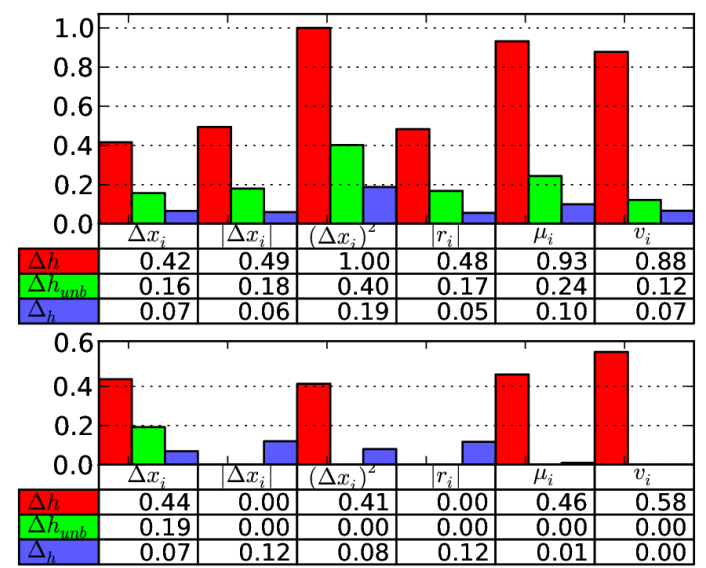

Fig. 9. The same as in Fig. 8 but for Nikkei225 index in two adjacent time windows since Nov. 27, 2003 till Dec. 19, 2007 and since Dec. 20, 2007 till Jan. 30, 2012.

from $\Delta h$ spread in particular. The observed multifractal structure of series is often completely hidden in a multifractal bias. It applies particularly to more complicated nonlinear transformations, including the commonly used volatility of the $v_{i}$ or $\mu_{i}$ types.

One must keep in mind that multifractal profile drawn in a bias ribbon is undistinguishable from the one caused by FSE or nonlinear transformations coupled with FSE, and only the values exceeding this bias have statistical significance of the multifractality present originally in primary data. This problem is best summarized in Figs. 8, 9 where both values $\Delta h_{\text {obs }}$ (red) and $\Delta h_{\text {unb }}$ (green) calculated for each of the cases in Figs. 5, 6 are shown, respectively.

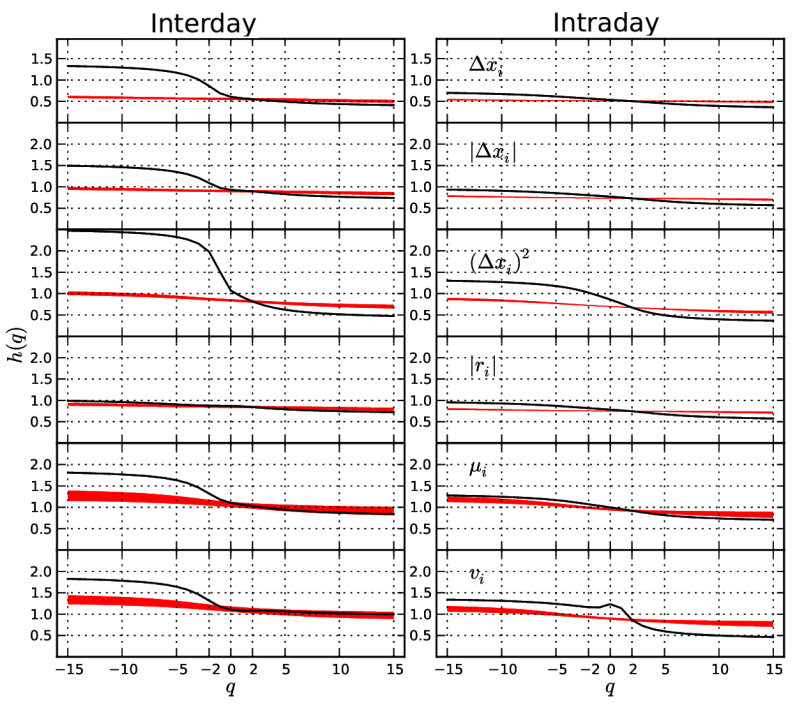

Fig. 10. Comparison between multifractal profiles (black curves) for intraday and interday data of WIG index shown with corresponding total multifractal bias (red ribbon areas). The multifractal profiles for interday data were calculated accounting all available closure quotations in period 1991-2012. The intraday results have been divided into segments of equal length corresponding to the amount of all available historical closure data points $L=4742$ in the same period and an average multifractal profile over such segments is shown. Statistical uncertainty is not indicated in the right column to make the plots in the current figure more readable. Statistical uncertainty bars are shown in Fig. 11.

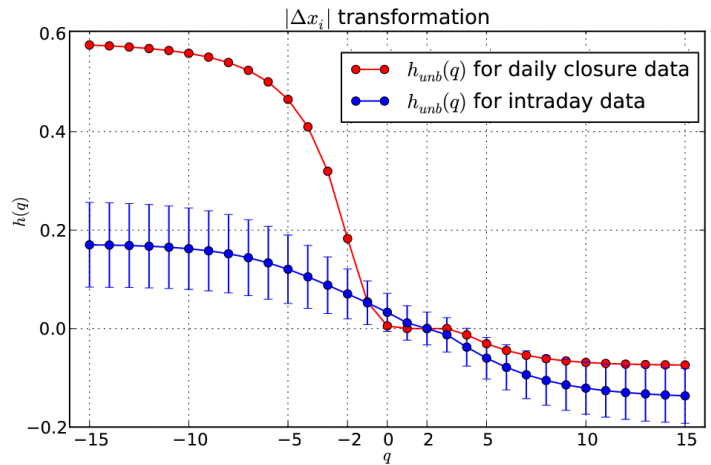

Fig. 11. Comparison of unbiased multifractal profiles $h_{\text {unb }}(q)$ for absolute increments $\left|\Delta x_{i}\right|$ of intraday and interday series from WIG data as in Fig. 10. The standard deviations of the intraday results are shown as error bars calculated over ensemble of 113 non-overlapping segments of length $L=4742$ each.

The next problem considered in the context of multifractal bias concerns an influence of time resolution. The multifractal structures of high- and low-frequency quotations from the main index of Warsaw stock exchange - WIG are compared together. The chosen sets of data were: 


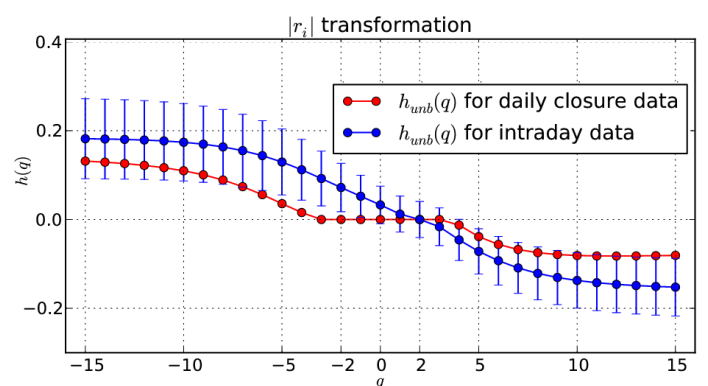

Fig. 12. The same as in Fig. 11 but for absolute returns $\left|r_{i}\right|$ of WIG data.

- interday - complete historical 4742 daily quotations in the time period from 16 Apr. 1991 till 31 Jan. 2012,

- intraday - series of 535846 one minute quotations in the time period from 20 Feb. 2003 till 20 Oct. 2008.

TABLE II

Comparison of biased and unbiased multifractal spread respectively for low- and high-frequency quotations from WIG index. The table shows evaluation of Eq. (10) for $h(q)$ profiles visible in Fig. 10.

\begin{tabular}{c|c|c|c|c|c|c}
\hline \hline \multicolumn{7}{c}{ Interday } \\
\hline & $\Delta x_{i}$ & $\left|\Delta x_{i}\right|$ & $\left(\Delta x_{i}\right)^{2}$ & $\left|r_{i}\right|$ & $\mu_{i}$ & $v_{i}$ \\
\hline$\Delta h_{\text {obs }}$ & 0.92 & 0.75 & 2.00 & 0.26 & 0.97 & 0.83 \\
$\Delta h_{\text {unb }}$ & 0.87 & 0.70 & 1.77 & 0.22 & 0.83 & 0.65 \\
\hline \multicolumn{7}{c}{ Intraday } \\
\hline & $\Delta x_{i}$ & $\left|\Delta x_{i}\right|$ & $\left(\Delta x_{i}\right)^{2}$ & $\left|r_{i}\right|$ & $\mu_{i}$ & $v_{i}$ \\
\hline$\Delta h_{\text {obs }}$ & 0.34 & 0.36 & 0.94 & 0.38 & 0.57 & 0.88 \\
$\Delta h_{\text {unb }}$ & 0.32 & 0.32 & 0.68 & 0.34 & 0.34 & 0.65
\end{tabular}

The intraday series was created in such a way that differences between close and open index values, coming from market animation, were not taken into account. To compare influence of FSE in the series we have had to equalize their lengths. The intraday series containing originally 535846 data points was cut into 113 non-overlapping sequences of length 4742. Eventually, the multifractal profiles calculated for these 113 sequences were averaged and compared with the interday multifractal results in Fig. 10. The standard deviation from the average is plotted in Figs. 11, 12. These results show that the main difference is visible for negative values of the $q$-moments. They reveal multiscaling only for small fluctuations in low-frequency daily quotations for primary data as well as for almost all transformations except $\left|r_{i}\right|$. The multifractal bias present in Fig. 10 as red-ribbon is not negligible and grows for more complicated transformations (4), (5). This fact has already been noticed in preceding figures. The exact values of $\Delta h_{\mathrm{obs}}$ and $\Delta h_{\mathrm{unb}}$ are gathered in Table II. The case of absolute returns transformation (3) is exceptional, because their unbiased multifractal profiles of interday and intraday series are statistically undistinguishable. One gets no evidence of multifractality present in initial data $\Delta x_{i}$ looking just at the observed (biased) multifractal profiles of absolute returns. The multifractal effect generated by nonlinear $\left|r_{i}\right|$ transformation makes strong background in comparison with unbiased multifractality of absolute returns shown in Fig. 12. It is not a case for other volatile transformations on WIG data (see Figs. 10, 11).

\section{Introducing new measure of unbiased multifractality}

The results of the previous section convince that a traditional measurement of multifractality level in MF-DFA based entirely on the $\Delta h$ spread may be misleading. Often the biased signal indicates the presence of apparent multifractal properties in time series while the unbiased spread $\Delta h_{\text {unb }}$ does not confirm this. There are frequent situations, particularly for a more complex nonlinear transformation of real data, where the $h(q)$ profile is not monotonically decreasing and extends partially or completely outside the admissible multifractal bias level $h(q)_{\mathrm{b}}$ despite one or both of the edges $h^{ \pm}$of the multifractal profile hidden in the bias domain (see e.g. most cases of $h(q)$ profiles in Figs. 5, 6). Therefore we propose an alternative measure of multifractal properties in generalized Hurst exponent approach which takes into account the behavior of the multifractal profile $h(q)$ as a whole for all $q$-th order moments. A natural extension of the $\Delta h$ spread in this novelization is to cumulate contributions to this spread from all $q$ moments. We define the "cumulative" unbiased multifractal spread $\Delta_{h}$ as

$$
\Delta_{h}=\frac{1}{Q} \int_{-Q}^{Q}\left|h_{\mathrm{obs}}(q)-h_{\mathrm{b}}(q)\right| \mathrm{d} q,
$$

where $Q=q_{\max }$ is the maximal considered moment in MF-DFA (in this article $Q=15$ was assumed).

In other words the cumulative unbiased spread is the cumulated and normalized distance between the unbiased observed multifractal profile $h_{\text {obs }}(q)$ and the multifractal bias $h_{\mathrm{b}}(q)$. Obviously $\Delta_{h} \geq 0$ and even if $\Delta h_{\mathrm{unb}}=0$ one may get $\Delta_{h}>0$ for a particular case.

The results of the new measure obtained for all the cases in Fig. 5 and Fig. 6 were added as the third bar (blue) in Fig. 8 and Fig. 9. It should be stressed that in many cases when $\Delta h_{\text {unb }}$ measurement is equal to zero and therefore is not indicative for multifractality present in the system, the newly proposed measuring technique $\Delta_{h}$ does confirm existence of multifractality (see e.g. the bottom parts: of Fig. 8, 9 - transformations (1)-(4) and the corresponding full $h(q)$ plots in Figs. 5, 6).

\section{Concluding remarks}

The concept of multifractal bias effects was introduced in this paper. This bias comes from the finite length of investigated data amplified by the long-term memory present in time series and from eventual nonlinear transformations applied to initial (primary) series of 
data. We have shown that such transformations seriously modify an initial FSE multifractal bias. Generally, the more complex nonlinear volatility transformation is carried out, the greater the level of multifractal bias is registered in the final outcome. This result was confirmed for data mined from three different markets, each from different part of the world. A proper identification of such biased effects is crucial in an unambiguous determination of the multifractality level in the investigated data. It was shown that even for clearly multifractal series (binomial cascade algorithm) the monofractal bias can take up to $\approx 35 \%$ (see Fig. 3, transformation (5)) of the whole multifractal spread.

For real series coming from very complex example of stock market indices, one finds that even for time series before transformation the multifractal structure of data is strongly biased. The investigation of low- and high-frequency quotations show that multifractal bias plays an important part for both of them (see Table II). The comparison of these sets of data reveals richer multiscaling properties for daily data, but only in the range of negative $q$ values, i.e. for very small fluctuations. The unbiased multifractal profiles of interday and intraday series for absolute returns in WIG index were found statistically undistinguishable. Moreover, the observed (biased) multifractal profiles of absolute returns are almost entirely formed by nonlinear $\left|r_{i}\right|$ transformation. This effect is so strong that it relatively suppresses multifractality in initial data thus making the $\left|r_{i}\right|$ transformation unappropriate for discovery of multiscaling properties in primary series $\Delta x_{i}$. Such a suppression does not happen in case of other volatile transformations on WIG data both interday and intraday quotes.

The analysis of adjacent windows shows clearly different multiscaling features for steady periods and very dynamic ones with extreme events. The investigated examples of the "Black Monday" crash in 1987 and the fall 2008 crash on Nikkei225 index convince that complexity of stock market time series flees out from the traditional measurements of multifractality based only on the spread of multifractal profile. Hence, a new approach, called "cumulative" unbiased multifractal spread $\Delta_{h}$ was proposed. The provided examples prove that new method is useful, especially in very dynamical periods of market evolution, when the simpler predecessor cease to work (see e.g. the right parts of Fig. 8 - transformations $\left|\Delta x_{i}\right|,\left(\Delta x_{i}\right)^{2}$, $\left|r_{i}\right|, \mu_{i}$ and Fig. 9 - transformations $\left.\left|\Delta x_{i}\right|,\left(\Delta x_{i}\right)^{2},\left|r_{i}\right|\right)$.

We observed also that more complex transformations of initial data, like $\mu_{i}$ and $v_{i}$ carry more multifractal bias which may make them less reliable for practical purposes when a search for multifractal properties of primary data is undertaken. One must also keep in mind that red bars in Figs. 8 and 9 show the biased multifractal spread and are unreliable because they carry the summarized effect of applied nonlinear transformations together with FSE amplified by the presence of long-term memory.

\section{References}

[1] M.S. Taqqu, V. Teverovsky, W. Willinger, Fractals 3, 785 (1995).

[2] Fractals in Science, Eds. A. Bunde, S. Havlin, 2nd ed., Springer, Berlin 1996.

[3] H.E. Hurst, Trans. Am. Soc. Civ. Eng. 116, 770 (1951).

[4] B.B. Mandelbrot, J.R. Wallis, Water Resour. Res. 5, 321 (1969).

[5] J.W. Kantelhardt, E. Koscielny-Bunde, H.H.A. Rego, S. Havlin, A. Bunde, Physica A 295, 441 (2001).

[6] J.W. Kantelhardt, in: Encyclopedia of Complexity and Systems Science, (Fractal and Multifractal Time Series), Ed. R.A. Meyers, Springer, Berlin 2009, p. 3754 .

[7] J.F. Muzy, E. Bacry, A. Arneodo, Phys. Rev. Lett. 67, 3515 (1991).

[8] J.W. Kantelhardt, S.A. Zschiegner, E. Koscielny-Bunde, S. Havlin, A. Bunde, H.E. Stanley, Physica A 316, 87 (2002).

[9] C.-K. Peng, S.V. Buldyrev, S. Havlin, M. Simons, H.E. Stanley, A.L. Goldberger, Phys. Rev. E 49, 1685 (1994).

[10] C.-K. Peng, S. Havlin, H.E. Stanley, A.L. Goldberger, Chaos 5, 82 (1995).

[11] P. Oświęcimka, J. Kwapień, S. Drożdż, Phys. Rev. E 74, 016103 (2006).

[12] L. Telesca, V. Lapenna, M. Macchiato, Physica A 354, 629 (2005).

[13] L. Telesca, V. Lapenna, Tectonophysics 423, 115 (2006).

[14] M.S. Movahed, F. Ghasemi, S. Rahvar, M.R.R. Tabar, Phys. Rev. E 84, 021103 (2011).

[15] P.H. Figueiredo, E. Nogueira Jr., M.A. Moret, S. Coutinho, Physica A 389, 2090 (2010).

[16] S. Dutta, J. Stat. Mech. 12, P12021 (2010).

[17] I.T. Pedron, J. Phys. Conf. Series 246, 012034 (2010).

[18] F. Liao, Y.-K. Jan, J. Rehab. Res. Develop. 48, 787 (2011).

[19] Y. Leung, E. Ge, Z. Yu, Ann. Assoc. Am. Geograph. 101, 1221 (2011).

[20] G.R. Jafari, P. Pedram, L. Hedayatifar, J. Stat Mech., P04012 (2007).

[21] P. Oświęcimka, J. Kwapień, I. Celińska, S. Drożdż, R. Rak, arxiv/1106.2902v1 [physics.data-an].

[22] F.A. Hirpa, M. Gebremichael, T.M. Over, Water Resour. Res. 46, W12529 (2010).

[23] M. Pasquini, M. Serva, Econ. Lett. 65, 275 (1999).

[24] K. Ivanova, M. Ausloos, Physica A 265, 279 (1999).

[25] A. Bershadskii, Eur. Phys. J. B 11, 361 (1999).

[26] N. Vandewalle, M. Ausloos, Eur. Phys. J. B 4, 257 (1998).

[27] A. Bershadskii, Physica A 317, 591 (2003).

[28] T. Di Matteo, T. Aste, M.M. Dacorogna, Physica A 324, 183 (2003).

[29] K. Matia, Y. Ashkenazy, H.E. Stanley, Europhys. Lett. 61, 422 (2003) 
[30] P. Oświęcimka, J. Kwapień, S. Drożdż, Physica A 347, 626 (2005).

[31] J. Kwapień, P. Oświęcimka, S. Drożdż, Physica A 350, 466 (2005).

[32] Z. Chen, P.Ch. Ivanov, K. Hu, H.E. Stanley, Phys. Rev. E 65, 041107 (2002).

[33] D. Grech, G. Pamuła, Acta Phys. Pol. A 121, B34 (2012).

[34] D. Grech, G. Pamuła, arxiv/1108.1951v2.

[35] G. Pamula, D. Grech, in preparation.

[36] S. Drożdż, J. Kwapień, P. Oświęcimka, R. Rak, Europhys. Lett. 88, 60003 (2009), arxiv/0907.2866 [physics.data-an].

[37] Wei-Xing Zhou, arxiv/0912.4782v [q-fin.ST].

[38] J. Ludescher, M.I. Bogachev, J.W. Kantelhardt, A.Y. Schumann, A. Bunde, Physica A 390, 2480 (2011).

[39] A.Y. Schumann, J.W. Kantelhardt, Physica A 390 , 2637 (2011).
[40] D. Gulich, L. Zunino, Physica A 391, 4100 (2012).

[41] T. Kalisky, Y. Ashkenazy, S. Havlin, Phys. Rev. E 72, 011913 (2005).

[42] H.A. Makse, S. Havlin, M. Schwartz, H.E. Stanley, Phys. Rev. E 53, 5445 (1996).

[43] J. Feder, Fractals, Plenum Press, New York 1988.

[44] R.C. Altman, "The Great Crash 2008", Foreign Affairs, (Jan./Feb. 2009), p. 1.

[45] G.M. Hodgson, Cambr. J. Econ. 33, 10205 (2009).

[46] N. Vandewalle, M. Ausloos, Ph. Boveroux, A. Minguet, Eur. Phys. J. B 9, 355 (1999).

[47] D. Sornette, R. Woodard, Wei-Xing Zhou, Physica A 388, 8 (2009).

[48] E. Carnecki, D. Grech, G. Pamuła, Physica A 387, 6801 (2008). 